\title{
Toward the harmonization of immune monitoring in clinical trials: Quo vadis?
}

\author{
C. M. Britten · S. Janetzki $\cdot$ S. H. van der Burg $\cdot$ \\ C. Gouttefangeas $\cdot$ A. Hoos
}

Received: 23 June 2007 / Accepted: 17 July 2007 / Published online: 25 August 2007

(C) Springer-Verlag 2007

\section{Introduction}

A constantly increasing number of cancer immunotherapies are being investigated in clinical trials but no reliable biomarkers to predict clinical benefit currently exist. For some cancer types, biomarkers have proven to be meaningful predictors of patient outcomes (e.g., BCR-ABL in Chronic Myeloid Leukemia or PSA in prostate cancer) and were established as routine tools. As effects of cancer immunotherapy are mediated through the immune system, immune

C. M. Britten and S. Janetzki both authors contributed equally to this work.

This commentary refers to the two original articles "The CIMTMonitoring panel: A two-step approach to harmonize the enumeration of antigen-specific CD8+ T lymphocytes by structural and functional assays" by C. M. Britten et al. and "Results and Harmonization Guidelines from two large-scale international Elispot proficiency panels conducted by the Cancer Vaccine Consortium (CVC/SVI)" by $\mathrm{S}$. Janetzki et al.

\section{M. Britten ( $\square)$}

Department of Immunohaematology and Blood Transfusion, Leiden University Medical Center, Leiden, The Netherlands e-mail: cebritten@web.de

S. Janetzki

ZellNet Consulting, Inc., Fort Lee, NJ, USA

S. H. van der Burg

Department of Clinical Oncology,

Leiden University Medical Center, Leiden, The Netherlands

C. Gouttefangeas

Department of Immunology,

University of Tuebingen, Tuebingen, Germany

A. Hoos

Bristol-Myers Squibb, Wallingford, CT, USA responses may act as natural biomarkers for clinical efficiency if the right factors can be reliably measured. Following this concept, cancer immunotherapy trials over the last decade have often included measures of tumor-specific cellular immune responses as endpoints to identify reliable surrogates for clinical benefit. Substantial efforts were invested throughout the immunotherapy field in setting up suitable cellular immune assays. However, to date, data from clinical trials do not consistently show that immune responses are correlated with clinical endpoints [1]. This lack of correlation is partially interpreted as a consequence of the high variability in assay results, due to the lack of assay standardization, validation and harmonization across laboratories. If harmonization of immune assays can be achieved, assays can be tested as surrogate endpoints in clinical trials, substantially accelerate the development of immunotherapeutic agents and, in addition, offer a rationale to pre-select groups of patients with high probability to benefit from subsequent immunotherapy.

A plethora of different immunological assays to monitor antigen-specific $\mathrm{T}$ cell responses are available, and some are used by a majority of laboratories. Prime examples are the ELISPOT assay, intracellular cytokine staining (ICS), MHCpeptide multimer staining for detection of antigen-specific $\mathrm{CD}^{+}$and increasingly for $\mathrm{CD}^{+} \mathrm{T}$ cells and proliferation assays based on carboxyfluorescein succinimidyl ester (CFSE)-labelling. Additional functional assays that have been introduced more recently are based on CD154 up-regulation on activated antigen-specific $\mathrm{CD} 4^{+} \mathrm{T}$ cells or the detection of CD107a or cytotoxicity related molecules like perforin or granzyme in $\mathrm{CD}^{+} \mathrm{T}$ cells. For each of these assays, the use of different protocols that have evolved over time translates into a wide range of performances. Differences in the interpretation of the results obtained make comparisons of published data even more complex. This has led to lack of 
comparability as well as doubts about the integrity of the results that were obtained and published worldwide [2].

Recent developments clearly suggest that "proper" immunomonitoring will have to encompass the parallel use of several tests, which together assess the frequency, function and homing capacity of induced/stimulated $\mathrm{T}$ cells present in the circulation and preferentially also locally within the targeted tissue. In view of the influence of both natural and adaptive regulatory $\mathrm{T}$ cells on clinical outcome, immunomonitoring should also include screening for these cells. Only the combined use of several techniques may lead to a valid set of surrogate markers for specific immune interventions that allows clinical decisions or optimization of immunotherapeutic strategies. A related proposal was made by a community-wide consensus workshop on clinical trials with cancer vaccines, in which criteria for the use of immune assays were recommended including at least two validated assays to be used in parallel [3].

There is also a surprising disparity between the substantial financial and logistic efforts invested in reagents and man hours to develop immune monitoring techniques and the comparatively minor investment made to date in actually validating and standardizing the available techniques within and between laboratories. This has to be critically re-evaluated as efforts put in the adequate harmonization of assays will support the investments already made. They are also a pre-requisite to meet regulatory requirements on assay standards prior to them being considered as surrogate endpoints for clinical trials. This affects academic institutions and industry partners alike and may reach beyond cancer into the fields of autoimmune and infectious diseases.

\section{How can the immunotherapy community best achieve immune assay harmonization?}

The goals of harmonization are to identify crucial protocol choices that influence the outcome of an applied assay, and to standardize those choices across laboratories throughout the entire field for optimal performance and consistent results. Recent activities in the immunotherapy field indicate that there is an increasing awareness of the necessity of such harmonization and assay validation [4-8]. In parallel to intra-laboratory standardization, leading to standard operating procedures (SOP) for a given work setting, large comparative studies between laboratories with different protocols are required to identify and standardize crucial aspects in the protocols used and provide external validation. Such studies bear various logistical, organisational, and financial challenges. A well-coordinated centralized effort is necessary to accomplish this goal. Proficiency panels conducted by community-spanning organizations are a suitable answer.
Over the last 2 years, two non-profit organizations in the cancer immunotherapy field, the Cancer Vaccine Consortium (CVC) of the Sabin Vaccine Institute and the Cancer Immunotherapy (CIMT) monitoring panel have independently initiated large international proficiency panels. Their findings are now being published in two "technical focus articles" in this issue of CII [9, 10]. Both studies collected specific protocol information from participating laboratories and identified various protocol variables, which clearly can influence the assay outcome. Some of them appear to be "common sense" decisions, but are still neglected by a part of the laboratories in the immunotherapy field. Most importantly, the findings reported are concordant in both studies, and no contradiction in results is imminent. This is particularly relevant when taking into account that both panel initiatives chose a different experimental design. The need to increase the awareness for harmonization initiatives and external validation of assay performance is one of the general common findings of both initiatives. Specific recommendations arising for the ELISPOT from both CVC and CIMT panels are based on the importance of accurate and optimal number of viable cells added per well, which determine the sensitivity of the assay. These recommendations include a resting-phase of cells, the use of automated cell counters allowing the determination of apoptotic and dead cell fractions, and an optimal number of cells per well. Another crucial variable is the unspecific stimulation of cells, which can prevent detection of low frequency $\mathrm{T}$ cell response and demands the use of autologous APC as well as critical selection of serum sources. Appropriate plate evaluation strategies and training status of personnel further influence the assay outcome. These findings offer first harmonization guidelines that could instantly be accepted by a broad user base. Further assay optimization can be accomplished by conducting proficiency panels that specifically focus on the incorporation of identified crucial assay variables.

Historically, the first inter-laboratory testing reports for ELISPOT were published 7 years ago [11]. Subsequently, some guidelines resulting from a workshop for assay performance were published about 2 years later [12]. An important proficiency panel program was initiated by the NIAID (National Institute of Allergy and Infectious Diseases), Division of AIDS, for the HIV vaccine field, which served as a first model for the CVC panel program [4]. All these initiatives have certainly attracted attention of the scientific community but have so far not led to broadly accepted standards for immune monitoring. However, through the recent proficiency panels of the CIMT and CVC, which are described in this issue of CII, a chance is given to harmonize assay criteria within widely applicable guidelines. 


\section{Cancer immunotherapy networks}

It is no co-incidence that both proficiency panels were conducted by community-wide organizations. The history of unsuccessful individual laboratory or small-scale efforts has lead to the idea of joining forces and of establishing nonprofit organizations that can serve as facilitators of the required initiatives in the United States and Europe. These organizations have large networks of members throughout the immunotherapy community. They (1) give regular updates on the scientific progress, (2) identify the bottlenecks and the various barriers to future development and (3) can facilitate the dialogue between the different stakeholders such as regulatory authorities, pharmaceutical and biotechnology companies, academic institutions, patients' advocacy groups and the public. Without these organizations the described proficiency panels would not have been possible.

The CVC as part of the Sabin Vaccine Institute initiated an Assay Working Group, which has conducted two largescale ELISPOT proficiency panels over the last 2 years with access for laboratories world-wide. These served not only as external validation programs for their participants (36 laboratories in panel I, and 29 laboratories in panel II), but also yielded first harmonization steps and guidelines to standardize and validate this assay as a reliable and widely acceptable tool for monitoring of immune responses in clinical trials.

The CIMT monitoring panel is a part of the Association for Immunotherapy of Cancer in Germany. It has until now initiated and completed two phases of an inter-laboratory testing project with focus on ELISPOT and tetramer staining (12 laboratories in panel phase I and 13 laboratories in panel phase II). The initiative is open for all labs with focus on immunomonitoring. A two-step approach was established that facilitates the systematic search for protocol variables that are predictive for high performance in the assay.

The breadth and size of both programs have the potential to help shape immune assay standardization and validation, and serve the ultimate goal of assay harmonization. Collaboration between both groups has proven meaningful for this first round of testing and is planned to increase for future activities.

\section{Perspective}

The conducted proficiency panels represent the largest inter-laboratory testing projects in this field to date. They provide first harmonization guidelines for ELISPOT assay and Tetramer staining. In addition, they demonstrate that international collaboration on this scale driven by nonprofit organizations is feasible and forms the basis for expanded future initiatives. Consequently, new Proficiency
Panels are now being started or are already under way. They include for CVC: ELISPOT, Tetramer, ICS and CFSE-labelling, and for CIMT: Elispot, Tetramer, ICS. Presentations during the coming annual meetings of both organizations will focus on recent developments and new technologies in the field. First guidelines for validation and training beyond this publication as well as for assay acceptance are in preparation. Additional organisational structures are now being established to offer the possibility of regular participation in different panels to the increasing number of laboratories world-wide that are seeing the value in assay harmonization. Aims are to find standards for training of staff, requirements for handling and test performance and for generating, measuring and reporting of data in broadly available technologies.

With the larger picture in mind, harmonization of these assays may provide the immunotherapy community with valuable surrogate markers for developing new therapies in clinical trials. This may ultimately help improving the clinical success of immunotherapies and bring them to patients more expeditiously.

\section{References}

1. Romero P, Cerottini JC, Speiser DE (2004) Monitoring tumor antigen specific T-cell responses in cancer patients and phase I clinical trials of peptide-based vaccination. Cancer Immunol Immunother $53: 249$

2. Rosenberg SA, Yang JC, Restifo NP (2004) Cancer immunotherapy: moving beyond current vaccines. Nat Med 10:909

3. Hoos A, Parmiani G, Hege K, Sznol M, Loibner H, Eggermont A, Urba W, Blumenstein B, Sacks N, Keilholz U, Nichol G (2007) Cancer vaccine clinical trial working groupA clinical development paradigm for cancer vaccines and related biologics. J Immunother 30:1

4. Cox JH, Ferrari G, Kalams SA, Lopaczynski W, Oden N, D’Souza MP (2005) Elispot collaborative study group results of an ELISPOT proficiency panel conducted in 11 laboratories participating in international human immunodeficiency virus type 1 vaccine trials. AIDS Res Hum Retroviruses 21:68

5. Janetzki S, Cox JH, Oden N, Ferrari G (2005) Standardization and validation issues of the ELISPOT assay. Methods Mol Biol 302:51

6. Hanekom WA, Dockrell HM, Ottenhoff THM, Doherty TM, Fletcher H, McShane H, Weichold FF, Hoft DF, Parida SK, Fruth UJ (2007) Immunological outcomes of novel TB vaccine trials: WHO panel recommendations (submitted)

7. Speiser DE, Pittet MJ, Guillaume P, Lubenow N, Hoffman E, Cerottini JC, Romero P (2004) Ex vivo analysis of human antigenspecific CD8+ T-cell responses: quality assessment of fluorescent HLA-A2 multimer and interferon-gamma ELISPOT assays for patient immune monitoring. J Immunother 27:298

8. Comin-Anduix B, Gualberto A, Glaspy JA, Seja E, Ontiveros M, Reardon DL, Renteria R, Englahner B, Economou JS, GomezNavarro J, Ribas A (2006) Definition of an immunologic response using the major histocompatibility complex tetramer and enzymelinked immunospot assays. Clin Cancer Res 12:107

9. Britten CM, Gouttefangeas C, Schoenmaekers-Welters MJP, Pawelec G, Koch S, Ottensmeier C, Mander A, Walter S, Paschen A, Müller-Berghaus J, Haas I, Mackensen A, Køllgaard T, Thor 
Straten P, Schmitt M, Giannopoulos K, Maier R, Veelken H, Bertinetti C, Konur A, Huber C, Stevanović S, Wölfel T, Van der Burg SH (2007) The CIMT-Monitoring panel: A two-step approach to harmonize the enumeration of antigen-specific $\mathrm{CD} 8^{+} \mathrm{T}$ lymphocytes by structural and functional assays. Cancer Immunol Immunother. doi:10.1007/s00262-007-0378-0

10. Janetzki S, Panageas KS, Ben-Porat L, Boyer J, Britten CM, Clay TM, Kalos M, Maecker HT, Romero P, Yuan J, Kast WA, Hoos A, for the Elispot proficiency panel of the CVC immune assay working group (2007) Results and harmonization guidelines from two large-scale international Elispot proficiency panels conducted by the Cancer Vaccine Consortium (CVC/SVI) Cancer Immunol Immunother. doi:10.1007/s00262-007-0380-6
11. Scheibenbogen C, Romero P, Rivoltini L, Herr W, Schmittel A, Cerottini JC, Wölfel T, Eggermont AM, Keilholz U (2000) Quantitation of antigen-reactive $\mathrm{T}$ cells in peripheral blood by IFNgamma-ELISPOT assay and chromium-release assay: a four-centre comparative trial. J Immunol Methods 244:81

12. Keilholz U, Weber J, Finke JH, Gabrilovich DI, Kast WM, Disis ML, Kirkwood JM, Scheibenbogen C, Schlom J, Maino VC, Lyerly HK, Lee PP, Storkus W, Marincola F, Worobec A, Atkins MB (2002) Immunologic monitoring of cancer vaccine therapy: results of a workshop sponsored by the Society for Biological Therapy. J Immunother 25:97 